\section{Integrative Medicine \\ International}

\title{
Chaos Theory and Self-Organization Systems in Recovery Medicine: A Scientific Review
}

\author{
Alexander A. Khadartseva ${ }^{a}$ Valeriy M. Eskov ${ }^{b}$ \\ ${ }^{a}$ Medical Institute, Tula State University, Tula, and b Surgut State University \\ of the Khanty-Mansi Autonomous Okrug-Yugra, Surgut, Russia
}

\author{
Key Words \\ Synergetics $\cdot$ Chaos $\cdot$ Adaptation programs $\cdot$ Stress $\cdot$ Control
}

\begin{abstract}
In this review, the theoretical principles, which provide the possibility of correction of acute stress pathology, are formulated in terms of the theory of chaos and self-organization. We present the concepts of phase space, channels, wildcards, attractors, determinism, synergetics, etc. and give the basic compartment and cluster approach to complex systems. Additionally, we show the interdependence of system analysis and synthesis applied to the development of European and Oriental medicine. Furthermore, the importance of modulation in adaptation programs in the control of the functional systems of a human body, confirmed in the data submitted for 14 patents covering inventions that have led to 2 discoveries, is demonstrated.

(C) 2015 S. Karger AG, Basel
\end{abstract}

Theoretical positions should be expressed in terms of the theory of chaos and self-organization (TCS), so that their possible influence on the initial pathology in the internal organs can be determined with the objective of correcting it.

The tendency to formalize the information obtained from biological objects has led to the need for the extensive use of mathematical approaches and methods. The classical determinism of Laplace with its clear cause-and-effect relationships has been replaced by a stochastic approach. The methods of a stochastic, probabilistic analysis in biology and medicine that correspond to the laws of quantum mechanics in physics have been replaced by the concept of chaos. In the system 'determinacy-indeterminacy', determinism and chaos take on extreme positions. Determinism is true only within the context of certain agree- 
Khadartsev and Eskov: Chaos Theory and Self-Organization Systems in Recovery Medicine (Scientific Review)

ments. Chaos is continuous (Brownian motion of molecules etc.), but chaotic dynamics of biological systems become stochastic because of the abundance of redundant mechanisms and regulatory reverse relationships in the body (with one or another variation around the average values on the index). A deterministic approach can be used for the interpretation changes of a specific organism at short time intervals. There is a specific mathematical apparatus for describing each of the open systems (exchange with the environment, matter and energy). It has been established that it is possible to convert from determinism to a chaotic and stochastic system. This means that simple deterministic systems with a small number of components can generate incidental behavior which cannot be removed because a lot of information has not been collected. Accidents, which appear to have happened in this way, become known as chaos. In this sense, chaos is deterministic. But chaos itself can generate order. Thus, the Brownian motion of molecules is summarized as a stochastic ordering of organs and tissues followed by deterministic functioning of the organs and systems [1-3].

The interaction between chaos, determinism and stochastics has led to the development of synergetics as science. Synergetics (from the Greek 'synergetikos' - joint, acting in concert) is the science that studies the relationships between the elements of a structure (subsystems), which have been formed in open systems (biological, physical, chemical and others) due to intensive (streaming) exchange of matter and energy within an environment where conditions are not in equilibrium. In such systems, there is a consistent behavior of the subsystems with an increase in its orderliness but a decrease in entropy as a measure of chaos (approach of self-organization) [4].

Synergetics deals basically with the thermodynamics of nonequilibrium processes, the theory of random processes, and the theory of nonlinear oscillations and waves, etc. This term, first proposed by Hermann Haken, focuses modern scientific attention on the coordinated interaction of parts in the formation of a structure as a whole. Synergetics studies the processes of self-organization, appearance, maintenance of the sustainability and collapse in structures of various natures, and this is just the beginning. The TCS has been formulated. Researches in this area are carried out in many scientific fields, and each of these fields has its own terminology and uses methods peculiar to it $[5,6]$.

The TCS reflects the interdisciplinary relationship between the different sciences. The research objectives in the different scientific disciplines are represented by separate parts, which, as a result of cooperation, translated into specific spatial, temporal or functional structures. Thus, individual cells cooperate with each other to form the body. In this case, the cells are compartments that, in clusters, represent an entire organism. Cells form tissues and organs, which, in relation to the whole body, are the compartments, and in relation to the cell, they are clusters. Such structuring is not imposed from the outside. It is organized by the system itself due to the outside influx of substances and/or energy. A compartment-cluster theory about the organization of biological dynamical systems has been proposed [7]. The principles of self-organization have also been defined.

The conclusion of Hermann Haken about the occurrence of critical slowing down and critical functions close to points of instability like bifurcations is fundamentally important, particularly when studying how movements of the limbs are controlled. This indicates that movement coordination is a function of a self-organizing system, i.e. the brain. It is not the realization of a ready-made program such as the computer $[8,9]$.

The TCS explains the mechanisms of self-organization systems. They are far from being in equilibrium with conditions of instability. World order and harmony are the result of chaos consisting of opportunities and potentialities. But these opportunities in the chaos are organized, and chaos itself organizes them during the implementation process. All forms of development are present in chaos but in a minimized form. Adaptation systems became flexible and provide adaptation to the environment as a consequence of chaos. Chaos organizes the 
Khadartsev and Eskov: Chaos Theory and Self-Organization Systems in Recovery Medicine (Scientific Review)

transition to relatively stable forms of evolution and the switching cycles of operation systems $[10,11]$. Chaos is dualistic. It destroys and it creates. It is both virtual and real. The chaos is the abyss and consists of the folded opportunities. It realizes them and the order is set. This order is relative, being the temporal structure of the original chaos [12]. Chaos is relative, because it contains a measure of chaotic state and a measure of order. It is organized in this way. It is not a mess. It is a causal (deterministic), dynamical system. It integrates order and disorder in complex systems (complexity). This integration can be either virtually invisible to the researcher or it can be represented by a fractal structure.

In the process of studying and describing the chaotic behavior of complex nonlinear systems, synergetics uses a variety of methods. These include the definition of the order parameters or the study of the behavior of dissipative systems, attractors, evolution or the charting of bifurcations [13]. Internal and external noises (in the environment) are used by living organisms to highlight useful information. In terms of dynamical chaos in the phase space, the different attractors coexist. Noises provide random switching between them; in this case, the statistical characteristics of the attractor are determined not only by properties of biological dynamical systems (BDS) but also by the properties of the noise. Information about the phase and amplitude of the signal is encoded in the time intervals between switches [14].

Stochastic resonance is as a physical phenomenon, providing an increased response of a nonlinear system to a weak external signal in terms of a growth in the intensity of noise [15]. The optimal noise level provides a degree of order in the output signal and maximizes the integral characteristics in the output (the signal/noise ratio or the gain coefficient). In nonlinear dynamical systems, a number of modes of operation cannot be realized in the absence of noise.

The effects of weak and superweak fields and radiation (waves) are associated with the resonant processes. The energy of the external field can move into the energy of a mechanical vibration environment containing the acceptors of electromagnetic radiation. The patterns of order are formed during such interactions in a viscous and elastic environment. An external field 'harmonizes' individual fluctuations of the nanoscale acceptors, increasing the energy of oscillatory processes in the environment to values greater than the thermal noise. The energy of thermal vibrations in atoms is determined by the product of the Boltzmann constant $(\mathrm{K})$ at the average temperature of the system (T) - KT. This efficiency of weak and superweak impacts on physical and biological objects and processes has led to the introduction of the term 'dissipative resonance'. It is a process of self-organization in dissipative structures with quasiperiodic changes being made in the system parameters [16]. This is 'the phenomenon of the rise of oscillations under the action of external periodic forces due to the formation of the system structures of order' [17]. In this case, the selected resonance purity is missing. The system itself is able to 'tune in' on a particular external frequency, resonating with it. In complex systems, the phenomena of stochastic and dissipative resonance coexist alongside each other.

The theory of dissipative structures led to a thermodynamic approach of self-organization. The concept of synergetics proposed by Hermann Haken, where the structure is a condition resulting from the consistent behavior of a large number of particles, is interpreted by Ilya Prigozhin as a special concept which he calls 'dissipative structure'. In open systems, a homogeneous equilibrium can lose stability when exchanging streams of matter or energy with the environment and can irreversibly move in a nonuniform stationary state. These states are stable for small disturbances and are called dissipative structures.

Despite the fact that dissipation is associated with the concept of a scattering of various types of energy and attenuation movements and with a loss of information in open systems, dissipation is a source of structure formation - temporal, spatial and spatiotemporal (autowaves), in which cooperative phenomena are carried out. In the formation of dissipative structures, the role of the collective, joint action is important, i.e. synergism. Dissipation is the 
source of BDS order in time and space. The goal of the search is to identify biological processes to the phenomena in which the condition is outside of the stability of the thermodynamic branch, 'far from equilibrium and instability', which provide a new state of matter that occurs with the flow of free energy [18].

Any BDS consists of concepts: condition (essential information about the system) and dynamics (rules describing the evolution of the system over time). The evolution of the system is observed in the space of condition or phase space. Phase space is an abstract space in which coordinates are components of conditions, i.e. a degree of freedom system. BDS can develop both in continuous and in discrete time. In the first case, it is a flow and in the second case, it is a reflection. The functioning of systems of the human body during life corresponds to the flow and human existence (birth, death) - to the reflection.

Thermodynamics of nonequilibrium (open, exchanging energy with the environment) systems, which include BDS, led to the emergence of the chaos theory and synergetics with the concepts of order parameters and the channels, the wildcards and the attractors. Phase space is based on many variables, by means of which our reality can be described. But in this space, there are also areas where a few parameters adequately reflect the many variables in the phase space as a whole and so help to understand and predict what is happening. When should these few variables be limited to predict with a certain accuracy the behavior of the entire system? Such areas in phase space or subspace are called the channels. A person's ability to allocate channels, to consciously act in terms of their limited options, led to the development of the whole of the previous science and medicine in particular. Under the channel, it is possible to build a theory, to calculate the options and to determine the strategy of influence. In synergetics, the variables characterizing the channels are called order parameters. The search of the channels by means of living systems, learning opportunities that neural networks used in computing practice, can be studied only an interdisciplinary science that integrates the data of psychology, neuroscience, management theory, neurophysiology and others. However, in real life, the channels (it is not known for what reasons) come to an end. The number of variables defining the functioning of the system increases (the slow variables are characteristic for the channels). The ability of the forecast is sharply limited, sudden changes occur.

In phase space, such places are called (in terms of synergetics) wildcard areas, when any random factor as a game element is crucial and affects the fate system or can even transfer the entire system abruptly to a different area of the phase space. In these areas the fast variables become decisive. Rules that describe such a leap are called wildcards. It is known that in a card game, the wildcard (the joker) is a card, to which a player can optionally assign the value of any card. This greatly increases the degree of uncertainty and the number of possible games. With regard to medicine, the areas away from the wildcards are available for therapeutic effects, and in the area of the wildcards only surgical and intensive care procedures are effective.

In the phase space, there are areas that characterize the system behavior after a long period of time and which are called the attractors (from the English 'to attract'). The simplest type of attractor has a fixed point (such as in the motion of a pendulum, for example), and a more complicated type of attractor has a limit cycle (in the form of a closed loop, for example, the beating of the heart), the attractor in the shape of a torus corresponds to complex, quasiperiodic motion. These are predictable attractors. There are also chaotic (strange) attractors acting as a pump, pumping with microscopic fluctuations and macroscopic in their manifestation. There is no predictability. Indeterminacy of initial measurement covers the entire attractor and makes predictions impossible. However, there are methods that make it possible to reproduce and reconstruct phase space and search for these chaotic (strange) attractors [19]. In humans, the central acceptor action that (according to Pyotr K. Anokhin) determines the behavior of the system is a multidimensional, chaotic and strange attractor which the BDS strives to reach by means of its self-organization. 
Khadartsev and Eskov: Chaos Theory and Self-Organization Systems in Recovery Medicine (Scientific Review)

The TCS describes the properties of systems that depend on the cooperative interaction of their elements without a reduction of their elements. These elements determine the order in which parameters operate, which in turn determines the behavior of the elements themselves. In this cycle what is primary and what is secondary cannot be defined because of so-called cyclic causality.

The chaos facilitates the transition of the system to a particular attractor at the bifurcation points, i.e. the branching of the ways of the evolution of the system. The fluctuations are the most pronounced in the period of the system instability. Moreover, microscopic fluctuations can cause the beginning of the evolution of the system in a new direction and change the entire macrosystem. Chaos also provides the possibility of combining simple structures to form complex structures and contributes to the harmonious development of the system and synchronization of the rate of flow processes inside. Chaos also provides the possibility of switching between various modes of system operation, thus preventing its destruction.

The interests of society and the development of civilization claimed synergetics and the concept of fractals. Economic crisis, natural disasters, social conflicts and war are part of the evolutionary crisis of humanity $[1,19]$. It is a transitional period of chaos, located between the old organization and the new order. Transitional processes in evolving systems are reflected in synergetics, as the theory of self-organization. Evolving systems are the essence of synergetics. The invariant core of such systems is structure preserving itself with various changes (in biology - DNA, the genome) [20]. When moving the system along the trajectory of the development, the control of this movement operates at the bifurcation points, and the orientation on one or other attractor is real.

We understand a systemic approach to be associated with the methods used in its study. As a result of the way in which it has developed science has become a large store of knowledge, but detailed information has been obtained mainly because of analysis (splitting of the whole). System analysis, which in recent years has become widely used in biological and medical science, involves the selection for study of the individual qualities and properties of an object. But for making decisions about control, it is necessary to take a holistic, systemic view of an object. The individual is not able to use a set of variables which, depending on the configuration of the set, may correspond to different channels, in which the system functions. Also, if its entry is into a system in the field of wildcards, the unpredictability of its behavior would lead to the complete indetermination of any external control.

This requires a systematic synthesis, which uses data from system analysis for the selection of a small number of parameters that define the vector of system development, and the relationship between these parameters. Processes that simplify and highlight the main point (averaging methods in mathematics or multilevel synthesis in programming) are already being used in science. Using a synergetic approach makes it possible to identify the parameters of an order or of a channel and to determine the mechanisms of their mutual transitions and to work with the wildcards. System synthesis explains how self-organization takes place in the space of capabilities, characteristics and the degree of freedom within a system and how individuals operate fuzzy sets by defining how they relate to the surrounding world. System synthesis determines the optimal structure of knowledge bases for the self-organization of knowledge and skills. The methods used for system analysis and synthesis will determine how the achievements of scientific and technological progress and the associated man-made disasters have affected the new state of the biosphere (and not only!) and will identify possible corrective measures. Achievements in biotechnology (decoding of the genome, cloning, etc.) need not only system analysis but system synthesis [19].

The conversion of an open living system to a chaotic norm by means of external control actions (treatment) is the process in a self-organizing system. This process can be described by the dynamics of the behavior of the state vector of the human body in the multidimensional 
phase space of states. Moreover, medicine does not work with all possible $m$-dimensional phase space, but it works with the order parameter, i.e. the signs $x_{i}$ (where $i=1,2, \ldots, \kappa$ ). In this case, the state vector of the human body is in the subspace of the states of the dimension $\kappa<m$, in which it is also possible to define the attractors of the norm (sanogenesis) and the attractors of pathology.

The development of the TCS in the third millennium is a necessary condition for the survival of humanity. A synergetic approach is the theoretical basis of integrative medicine, which combines traditional European medicine with ancient, including Eastern, medicine. Significant advances have been achieved by European medicine and the technologies used to further our understanding of the microcosm and the processes occurring in living organisms. In fact, hardly anyone would dispute the ground-breaking importance of Pyotr K. Anokhin's formulation of the theory of functional systems, which served as a basis for Norbert Wiener's modern description of cybernetics as a science. At the same time, ancient Eastern medicine has put the principles of system analysis and synthesis into in practice for thousands of years. Practical progress in diagnostic and treatment procedures has been difficult to explain from the perspective of European medicine and has often been rejected as 'unscientific'. However, in European medicine body functions have been widely studied using acupuncture to get information from biologically active points. The merging of European and Eastern medicine has been on a clinical basis. They are just two branches of the common ways of human cognition. This branching occurred for historical reasons. The 'European' roots of homeopathy going back to Samuel Hahnemann can also be seen in the principles of 'Zhen-Jiu therapy', where the small-dose stimulation of biologically active points based on the principle of 'similarity' causes significant systemic reactions of the body. This is confirmed by the results of research on biological effects of fields and radiation, showing that the controlling factor is a low-intensity electromagnetic influence. Therefore, in recent decades the concept of 'integrative medicine' operating results of traditional and alternative medicine (regardless of the view, which of them is traditional) has been formulated.

The fact that synergistics operates and controls the human body mechanisms has been established. In addition to the hypothalamic-pituitary-adrenal system that has been known since the time of Hans Selye, the role of the antagonist - the hypothalamic-pituitary-reproductive system - has also been established. The synergistic operation of these systems has been proved. The factor brain theory, based on the interactions of GABA and dopamine, has been confirmed. Their synergistic interactions have also been established [21].

In the opinion Anatoliy I. Grigoriev: 'During physiological and biological research, unique information about the life of the human body in extreme conditions has been received, new patterns of adaptation functional systems to altered gravity conditions have been discovered, new data about the peculiarities of the psychological reactions and health of astronauts during complex dynamic operations have been obtained and valuable experience of socialpsychological support of professional activity in the conditions of long isolation and stress has been gained. This is taken account the improvement of the system safety in long-term space flights' [22]. Further development in space medicine and biology requires new approaches to integration that are based on information coming from people who might reveal possible opportunities to correct potentially life-threatening situations or prevent them from developing.

Acute stress can be the trigger for first activating catatoxic adaptation programs, aimed at rejecting a stress agent. This is followed by the activation of the hypothalamic-pituitaryadrenal system. In cases where the stress agent exceeds the protective abilities of the body, the state vector of the human body turns abruptly to the mortal attractor. The death of the organism occurs. Another option is an abrupt transition to the activation of syntoxic adaptation programs, allowing the new channel stressor agents and humans to coexist, after which 
the hypothalamic-pituitary-reproductive system is activated. It is therefore possible chronic stress pathology. However, the potential of syntoxic adaptation programs and controlled systems is limited. When they are exhausted, a reactivation catatoxic adaptation program is real but is more likely to mean the death of the organism.

From positions of the TCS, these variations relate to the field of wildcards, so their predictability is extremely low. At the same time, the purposeful application of syntoxins could displace the human body into a new channel, with greater predictability of the course and the response to treatment of the effects of stress [21].

Internal diseases, as an artificially selected part of the diseases that afflict the human body due to an impaired control of functional systems (as a result of external or internal factors), lead to the development of the clinical picture of a disease. However, the symptoms of disease appear as an order parameter (in the terminology of the TCS). Our actions to control the body systems are therapeutic, preventive and rehabilitative measures.

The synergy effect is known during exposure on different levels of the control system (GABA-dopaminergic, hypothalamic-pituitary and hypothalamic-reproductive). The search for optimal combinations, levels and the duration for such control actions is the task of the doctor or the internist.

The methods of the TCS have been successfully used in the rehabilitation and the organization of activities in the treatment of ulcers $[23,24]$, chronic obstructive pulmonary disease [25], cardiorespiratory pathology [26, 27], diabetes [28, 29], biliary tracts [30], hypertension [31-33] and Reiter's disease [34]. They have also been used when developing the concept of personalized medicine $[35,36]$.

\section{Conclusion}

Despite a certain degree of conservatism of clinical medicine, it will be necessary to develop the TCS if a solution for biomedical problems is to be found. To achieve this, a common understanding of the terminology used in synergetics and TCS is important. There is a real possibility that the accumulated experience (mathematical apparatus of the TCS) could be adapted to meet the needs of clinical medicine, including the need to prevent situations involving acute and chronic stress. Methods of influencing the GABA-dopaminergic system of the brain as well as the hypothalamic-pituitary-adrenal and the hypothalamic-pituitaryreproductive system are available.

\section{References}

1 Eskov VM, Filatova OE, Khadartsev AA, Khadartseva KA: Fractal dynamics of human behavior-dimensional systems. Bull New Med Technol 2011;3:330-331.

2 Eskov VM, Khadartsev AA, Gudkov AV, Gudkova SA, Sologub LA: Philosophical and biophysical interpretation of life in the third paradigm. Bull New Med Technol 2012;1:38-41.

-3 Eskov VM, Khadartsev AA, Eskov VV, Filatova OE, Filatova DU: Chaotic approach in biomedicine: individualized medical treatment. J Biomed Sci Eng 2013;8:847-853.

4 Eskov VM, Khadartsev AA, Eskov VV, Gavrilenko TV, Filatov MA: Complexity is a special type of biomedical and social systems. Bull New Med Technol 2013;1:17-22.

5 Eskov VM: The Third Paradigm. Samara, LLC 'Ofort', 2011.

-6 Eskov VM, Khadartsev AA, Eskov VV, Filatova OE: Quantitative registration of the degree of the voluntariness and involuntariness (of the chaos) in biomedical systems. J Anal Sci Methods Instrum (JASMI) 2013;2:67-74.

7 Eskov VM: Compartmental-Cluster Approach in Studies of Biological Dynamical Systems (BDS). Part 1. Intercellular Interactions in Nanogenerators and Biomechanical Clusters. Samara, Publishing House 'ScientificTechnical Center', 2003. 
8 Haken H: Synergetics as a Bridge between the Natural and Social Sciences (transl. by Knyazeva EN); in Collection Synergetic Paradigm: Person and Society in Conditions of Instability. Moscow: Progress-Tradition, 2003, pp 106-123.

9 Haken H: Synergetics. Moscow, 'Mir', 1985.

10 Knyazeva EN: Balancing on the Edge of Chaos as a Creative Way to Update; in Collection Synergetic Paradigm: Person and Society in Conditions of Instability. Moscow, Progress-Tradition, 2003, pp 123-141.

11 Knyazeva EN, Kurdyumov SP: Synergetics as a New Worldview: Conversation with I. Prigozhin. Questions Philos 1992;12:3-20.

12 Filatova OE, Khadartsev AA, Eskov VV, Filatova DY: Uncertainty and Unpredictability - the Basic Properties of Systems in Biomedicine. Complexity Mind Postnonclassic 2013;1:67-82.

13 Prigozhin IR, Stengers I: Translation from English. Order out of Chaos: Man's New Dialogue with Nature. Moscow, Progress, 1986.

14 Anishchenko VS: Familiarity with Nonlinear Dynamics. Lectures Soros Professor. Moscow-Izhevsk, Institute of Computer Science, 2002.

15 Betsky OV, Kislov VV, Lebedev NN: Millimeter Waves and Living Systems. Moscow, Science Press, 2004.

16 Karnaukhov AV, Ponomarev VO: Dissipative Resonance - A New Class of Physical Phenomenon. Some Analytical Approaches to the Description. Biomed Technol Radioelectronics 2001;8:23-31.

17 Margolin VI, Zhabrev VA, Tupik VA: The Physical Basis of Microelectronics: Textbook for Students of High Education Institutions. Moscow, Publishing Center 'Academy', 2008.

18 Eskov VM, Burov IV, Filatova OE, Khadartsev AA: The Fundamentals of Bioinformatics Analysis of the Dynamics of Micro-Chaotic Behavior of Biological Systems. Bull New Med Technol 2012;1:15-18.

19 Malinetskiy GG: Chaos. Structures. Computational Experiment: Introduction to Nonlinear Dynamics. Moscow, Editorial URSS, 2000.

20 Voitsekhovich VE: Synergetic concept of fractals (social and philosophical foundations); in Collection Synergetic Paradigm: Person and Society in Conditions of Instability. Moscow, Progress-Tradition, 2003, pp 141-157.

21 Khadartsev AA, et al: Adaptogens in Medical and Biological Systems (Theory and Practice of Rehabilitation Medicine). Tula, LLC RIF 'INFRA' Moscow, 2005, vol 3.

22 Grigoriev AI, et al: Space Biology and Medicine (in 2 Volumes). Moscow, The Russian Federation State Research Center - Institute of Biomedical Problem of the RAS, 2001, vol 1: Medical Support of Long-Duration Missions.

23 Burmasova AV: Bioinformatics Analysis of the Parameters of the Organism of Patients with Peptic Ulcer of the Duodenum in the North Region (abstract); dissertation, Surgut, 2012.

24 Eskov VM, Karpin VA, Khadartsev AA, Burmasova AV, Eskov VV: Chaotic dynamics parameters of quasiattractors in the patients with peptic ulcer of the duodenum in conditions of medication and physiotherapeutic effects. Therapist 2013;5:63-71.

25 Rymogaeva NV: Bioinformatics Analysis of Secondary Prevention of Mixed Pathology with Cold Bronchial Obstructive Syndrome in the North (abstract); dissertation, Surgut, 2011.

26 Sokolova AA: Comparative Bioinformatics Analysis of the Parameters of the Cardio-Respiratory System in Adult Indigenous People and Newcomers of the Yugra (abstract); dissertation, Surgut, 2012.

27 Khadartsev AA, Eskov VM, Ushakov VF, Eskov VV: Program control of clinical examination and rehabilitation of patients with chronic obstructive pulmonary disease. Therapist 2013;7:4-15.

28 Dobrynina IY: System Analysis and Synthesis of Metabolic Disorders at Diabetes Mellitus Type 2 (abstract); dissertation, Tula, 2007.

29 Drozdovich EA: Bioinformational Analysis of Disorders of Autonomic Regulation of Cardiac Activity in Patients with Obesity and Diabetes Mellitus Type 2 in the Yugra (abstract); dissertation, Surgut, 2012.

30 Dudin NA: Bioinformatics Approach in the Assessment of Surgical Patients with Pathology of the Biliary Tracts (abstract); dissertation, Surgut, 2013.

31 Kondratyev AM: The Behavior of the State Vector of Patients with Injuries of the Lower Limbs and Arterial Hypertension in Phase Space (abstract); dissertation, Surgut, 2011.

32 Stepanova DI: Bioinformatics Analysis of Micro Chaotic Dynamics Parameters of Autonomic Regulation of the Inhabitants of the Surgut City with Chronic Diseases (abstract); dissertation, Surgut, 2012.

33 Shuvalova OI: Bioinformatics Analysis of Parameters of Patients with Arterial Hypertension in the Conditions of KMAO-Yugra (abstract); dissertation, Surgut, 2013.

34 Salyaeva LA: Method of Multidimensional Phase Spaces in the Assessment System Blood of Patients with Disease Reiter (in Conditions of the Surgut City) (abstract); dissertation, Surgut, 2011.

35 Eskov VM, Khadartsev AA, Kamenev LI: New Bioinformatics Approaches in the Development of Medicine from the Position of the Third Paradigm (Personalized Medicine - The Implementation of the Laws of the Third Paradigm in Medicine). Bull New Med Technol 2012;3:25-28.

36 Khadartsev AA, Fudin NA, Radtchcich IU: Physiological Bases of Visual Perception in the Training of Athletes from the Point of Synergetics. Bull New Med Technol 2012;2:17-20. 